\title{
Extended tumor control after dendritic cell vaccination with low dose cyclophosphamide as adjuvant treatment in patients with malignant pleural mesothelioma
}

Robin Cornelissen M.D., Ph.D. ${ }^{1}$; Joost P.J.J Hegmans Ph.D. ${ }^{1}$; Alexander P.W.M. Maat M.D. ${ }^{2}$; Margaretha E.H. Kaijen-Lambers ${ }^{1}$; Koen Bezemer'; Rudi W. Hendriks Ph.D. ${ }^{1}$; Henk C. Hoogsteden M.D. Ph.D. ${ }^{1}$; and Joachim G.J.V. Aerts M.D., Ph.D. ${ }^{1,3}$

${ }^{1}$ Department of Pulmonary Medicine, Erasmus MC Cancer Institute, Rotterdam, The Netherlands

2 Department of Cardio-Thoracic Surgery, Erasmus MC Cancer Institute, Rotterdam, The Netherlands

${ }^{3}$ Department of Pulmonary Medicine , Amphia Hospital, Breda, The Netherlands

\section{Corresponding author}

Joachim Aerts

Erasmus MC Cancer Institute

Department of Pulmonary Medicine,

Room H-Ee2218

P.O.-box 1738

3000 DR, Rotterdam, The Netherlands

Phone +31 107043697

Fax: +31 107044728 


\section{E-mail: j.aerts@erasmusmc.nl}

\section{Contributions}

Guarantor - RC, JPJJH \& JGJVA; Conception \& Design - JPJJH, JGJVA; Patient selection and patient data collection - RC and JGJVA; Patient treatment - RC, APWMM and JGJVA; Performing laboratory test and analysis - JPJJH, MEHK-L, KB; Interpretation of data - all authors; Manuscript Drafting and Revision - all authors; Final Approval - all authors.

\section{Financial Support}

This study was financially supported by Stichting Asbestkanker Rotterdam (www.asbestkanker.nl), Stichting Coolsingel, Stichting NutsOhra and the Mesothelioma Applied Research Foundation (MARF).

\section{Running title}

Consolidation immunotherapy in mesothelioma

\section{Descriptor number}

\subsection{Pleural Diseases/Mesothelioma}

\section{Total word count for the body of the manuscript}

$3495(\max 3500)$ 


\section{At a glance commentary}

Earlier we performed dendritic cell-based immunotherapy in a preclinical tumor model as well as in patients with mesothelioma. These studies showed that administration of whole tumor cell lysate-pulsed dendritic cells is feasible and that it can induce distinct immune responses and antitumor responses. However, some hurdles need to be overcome for improving clinical efficacy. Alleviation of the tumor-induced immune suppression could improve the efficacy of immunotherapy. In this study, 3 to 5 injections of tumor lysate-loaded monocytederived mature dendritic cells were administered to five patients after pemetrexed-cisplatin or pemetrexed-carboplatin treatment and five patients after induction chemotherapy followed by pleurectomy/decortication. Simultaneously, a metronomic dose of cyclophosphamide (CTX) was administered to these patients to investigate its potency to overcome immunosuppression by regulatory $\mathrm{T}$ cells (Treg). We showed that 1 -week of CTX significantly reduced the mean Treg percentage of total CD4 cells in peripheral blood, but an elevated Treg percentage initially was only found in a minority of patients. Furthermore, we demonstrated the safety and feasibility of this combination therapy in mesothelioma patients and found sustained tumor control.

\section{Online data supplement}

This article has an online data supplement, which is accessible from this issue's table of content online at www.atsjournals.org 


\section{Abstract}

Rationale: We demonstrated before that autologous tumor lysate-pulsed dendritic cell-based immunotherapy in patients with malignant pleural mesothelioma is feasible, well-tolerated, and capable of inducing immunological responses against tumor cells. In our murine model we found that reduction of regulatory $T$ cells with metronomic cyclophosphamide increased the efficacy of immunotherapy.

Objectives: To assess the decrease in number of peripheral blood regulatory $\mathrm{T}$ cells during combination therapy of low-dose cyclophosphamide and dendritic cell immunotherapy and to determine the induction of immunological responses with this treatment in mesothelioma patients.

Methods: Ten malignant pleural mesothelioma patients received metronomic cyclophosphamide and dendritic cell-based immunotherapy. During the treatment, peripheral blood mononuclear cells were analyzed for regulatory $\mathrm{T}$ cells and immunological responses.

Measurements and Main Results: Administration of dendritic cells pulsed with autologous tumor lysate combined with cyclophosphamide in mesothelioma patients was safe, the only side effect being moderate fever. Dendritic cell vaccination combined with cyclophosphamide resulted in radiographic disease control in eight of the ten patients. Overall survival was promising, with seven out of ten patients having a survival of $\geq 24$ months and two patients still alive after 50 and 66 months. Low-dose cyclophosphamide reduced the percentage of regulatory $T$ cells of total CD4 cells in peripheral blood from 9.43 (range 4.3426.10 ) to 4.51 (range $0.27-10.30$ ) after 7 days of cyclophosphamide treatment $(P=0.02)$. 
Conclusions: Consolidation therapy with autologous tumor lysate-pulsed dendritic cell-based therapy and simultaneously reducing the tumor-induced immune suppression is well-tolerated, and shows signs of clinical activity in mesothelioma patients.

\section{Abstract word count 246 (max 250)}

Keywords: immunotherapy, regulatory $\mathrm{T}$ cell, tumor microenvironment, immunosuppression, pleurectomy/decortication 


\section{Introduction}

Malignant pleural mesothelioma (MPM) is a highly lethal neoplasm with limited treatment options. Despite aggressive treatments with combinations of surgery, chemotherapy, and radiotherapy, it is almost inevitably accompanied with recurrences. Remarkably, however, long-term survivors do occur either with or without any treatment $(1,2)$. This may be explained by germline mutations, in which patients with BAP1 mutations recently were found to have an overall 7-fold increased long-term survival(3). Also, the role of the immune system in mesothelioma has provided new insights, which begin to explain the differences in the complex behavior of individual tumors and also uncover new possibilities for treatment(4).

Earlier we performed a phase I clinical trial using active immunotherapy in ten mesothelioma patients, who were treated with chemotherapy followed by three vaccinations of autologous tumor lysate-pulsed monocyte-derived dendritic cells (DC)(5). Results showed that these vaccines were well tolerated without systemic toxicity, and radiographic tumor responses were established. We were also able to detect distinct immune and cytotoxic $\mathrm{T}$ lymphocyte antitumor activity in the peripheral blood of these patients.

It is now well recognized that the efficacy of immunotherapy is influenced by the immunosuppressive environment created by the tumor(6). The presence of this immunosuppressive environment hampers attempts at harnessing the potency of the immune system. An increasing number of immunosuppressive factors and cells are reported in malignant diseases. We, among others, have 
established that regulatory T-cells (Tregs) play a major role in mesothelioma by contributing to an impaired T-cell function(7, 8).

Clinical studies have shown that low-dose cyclophosphamide (CTX) induces beneficial immunomodulatory effects in the context of active or adoptive immunotherapy by reducing the number of Tregs and their functionality $(9,10)$. In our murine model of mesothelioma we found that prolonged (metronomic) lowdose CTX augmented the anti-tumor effects of DC vaccines and increased survival via a reduction of the number of Tregs(11).

In the present study, we investigated whether these preclinical findings could be confirmed in patients. Ten patients with MPM who had previously been treated with chemotherapy or chemotherapy and debulking surgery were treated with CTX combined with DC-based immunotherapy as a consolidation/adjuvant treatment. The aim was to establish a reduction in the number of circulating Tregs by CTX and to determine the safety of immune therapy in these patients. We found that CTX combined with DC-immunotherapy was feasible and safe and that the number of Tregs in peripheral blood was reduced by a single cycle of CTX. Although in this report we will focus on the clinical results, the relevant immunological data will be presented. Significant increases in immune responsiveness were established, and overall survival was encouraging, with seven of the ten patients surviving $\geq 24$ months. Some of the results of the present study have been previously reported in the form of an abstract(12).

\section{Materials and Methods}




\section{Study design}

The study was approved by the institutional ethical committee of the Erasmus MC (MEC-2008-109) and the Central Committee on Research involving Human Subjects (CCMO; NL24050-000-08) as defined by the WMO (Medical Research Involving Human Subjects Act). Procedures followed were in accordance with the ethical standards of these committees on human experimentation and with the Helsinki Declaration of 1975 , as revised in 2008 . The study is registered at http://www.clinicaltrials.gov with identifier NCT01241682.

Patients with a suspicion of MPM and treatment-naive patients who had already been diagnosed with MPM were requested to give informed consent for isolating and storing pleural effusion cells if a medical indication was present to perform a pleural tap. Patients underwent standard treatment consisting of 4 to 6 courses of chemotherapy with either pemetrexed-cisplatin or pemetrexed-carboplatin. Response was assessed according to modified RECIST criteria(13).

Patients with either partial response or stable disease were evaluated for study treatment with DC-based immunotherapy. In case of progressive disease after chemotherapy, patients were excluded from participation and treated according to the clinicians' and patient's decision. It is general practice in our reference center to consider patients for a pleurectomy/decortication (P/D) only after completion of chemotherapy, in case of persistent complaints, e.g. dyspnea, related to tumor load. P/D is only performed in patients who are fit for operation and in whom a macroscopic debulking is considered feasible according to published guidelines. DC-based immunotherapy in combination with low dose CTX was planned 8 to 10 weeks after completion of the most recent cytoreductive therapy, i.e. either 
chemotherapy or surgery (figure 1). During treatment, blood and serum samples were taken from each participant at regular intervals. The blood samples were tested for immunological responses, liver function and renal function. In addition, the serum samples were screened for the development of auto-immunity. Overall survival was defined as survival after date of diagnosis. Final survival data was gathered in August 2015.

The study endpoints were (1) the reduction of regulatory T cells in the blood of patients after CTX, and (2), the safety and efficacy of the combination therapy consisting of DC vaccinations and CTX in MPM patients.

\section{Patient eligibility}

Signed written informed consent was obtained from each patient. At the time of diagnosis, patients were eligible for the study if they had an Eastern Cooperative Oncology Group (ECOG) performance status of 0 or 1 and acceptable vital organ functions. Patients were included if sufficient $\left(>150 \times 10^{6}\right)$ tumor cells could be obtained from the pleural effusion or tumor biopsy material at the time of diagnosis. Patients were eligible if their serum was tested negative for infectious agents (human immunodeficiency virus (HIV), human T-lymphotropic virus (HTLV) I\&II, hepatitis B virus (HBV), hepatitis C virus (HCV) and lues). Also, patients had to be negative for autoimmune disease and were tested for antinuclear antibodies (ANA), extractable nuclear antigens (ENA), and rheumatoid factors (RF). Delayed type hypersensitivity (DTH) skin tests were performed to investigate if the chemotherapeutic agents still exerted their influence on the patient's immune system. A solution of $3.75 \mathrm{Lf}$ of purified tetanus toxoid (NVI, Bilthoven, The 
Netherlands) was used as positive control and $25 \mu \mathrm{l} 0.9 \%$ saline as negative control. Reactivity to tetanus toxoid was measured in all the included patients 48 hours after subcutaneous injection (induration $>5 \mathrm{~mm}$ ) and was regarded as evidence for cellular immune competence.

\section{Dendritic cell vaccination and cyclophosphamide intake}

Eligible patients underwent immunotherapy based on loaded DCs in combination with low dose CTX. The tumor lysate that was used for loading of the DCs was obtained from pleural fluid in the non-surgical patients. In the surgical patients, the resection material was used for DC loading (see supplementary data).

Patients received at least three immunizations with mature DC loaded with autologous tumor lysate and $\mathrm{KLH}$ with a 2-week interval (Figure 1). Six and twelve months after the third DC vaccination, a revaccination was given to boost the immune system if enough dendritic cells were available $\left(4^{\text {th }} / 5^{\text {th }}\right.$ vaccination)(Table 2). Each immunization, consisting of $50 \times 10^{6}$ dendritic cells, was administered intradermally (i.d.) and intravenously (i.v.). Dosage was divided into $1 / 3$ administered i.d. in the forearm and $2 / 3$ administered i.v.. Blood pressure, body temperature, and oxygen saturation were constantly monitored till 2-h after the administration of vaccine therapy.

Patients were treated with 2 times $50 \mathrm{mg}$ tablet/day of CTX (Endoxan; Baxter B.V., Utrecht, The Netherlands) starting one week before vaccination to the day of every vaccination (followed by a week interval (figure 1)). The patients were asked to take the medication 2 hours after breakfast and dinner and to increase 
their fluid intake by drinking extra water or other non-caffeinated beverages during the day. Urine of patients was routinely checked for signs of hematuria.

\section{Results}

\section{Patients}

Between September 2009 and November 2011, 10 patients with advanced MPM and stable disease or response after chemotherapy were enrolled in the study. Patient characteristics are summarized in table 1. Nine patients commenced treatment within 6 weeks of diagnosis, and 1 patient opted for a delayed start of treatment (5 months). Five patients underwent an additional pleurectomy/decortication (table 2) before immunotherapy. One patient, patient 7 , was included in spite of previous treatment with several lines of therapy, because of a long interval between the last treatment and recurrence of the disease, for which renewed treatment was initiated. After pleurectomy/decortication was performed, he was treated in the study. The survival post-surgery is used in this study.

\section{Clinical response evaluation}

Radiographic responses performed after the third vaccination are shown in table 2. Of the 5 non-surgical patients, 4 had SD and 1 had $C R$ after DC therapy. In 3 of the surgically treated patients, the disease could not be evaluated, because surgery led to a macroscopic CR. However, 2 surgical patients presented with new lesions after DC therapy and therefore had PD. Therefore, in total, disease 
control (no remaining evaluable disease, CR, PR and SD) was achieved in 8 out of 10 patients. In addition, table 2 shows the overall survival of the patients with a follow up time of 6 years. Seven of the 10 patients had a survival of $\geq 24$ months (table 2 and figure 2). In August 2015, 2 patients were still alive, 1 of whom was in complete remission 66 months after diagnosis, and in the other the disease was very slowly progressive 50 months after diagnosis.

Figure 3 shows the CT scans of a surgically treated patient, a 35-year-old woman (patient 5) who presented with two pleural masses with no radiographic response to cisplatin-pemetrexed treatment. A successful pleurectomy/decortication was performed after induction chemotherapy, and pathological evaluation of the tumor revealed a largely vital tumor with only $20 \%$ signs of necrosis, compatible with the radiographic finding of SD after chemotherapy. Furthermore, the tumor extended into the resected margins. Five injections of adjuvant DC therapy were given and no disease recurrence has been seen yet, 66 months after initiation of treatment.

\section{Safety and toxicity}

None of the 10 patients withdrew from the study. There were no logistic problems or practical problems in the preparation process, so all vaccinations were given as planned. The safety and toxicity of the combination of a low dose CTX (orally) and tumor lysate-pulsed DCs injected intradermally and intravenously in patients with MPM were assessed. No related grade $>3$ toxicities were found in any of the patients. Table 3 presents a summary of the main adverse events not related to tumor progression. Injection of DCs was well tolerated without systemic toxicity, 
except for transient fatigue and low-grade fever on the day of the DC injection. These symptoms normalized after one day. After DC injection, a local skin reaction in the form of erythema without induration was seen in more than half of the subjects. Subsequent vaccinations (second and third) gave a quicker and increased induration and erythema in all patients, suggesting that some form of immunity was induced. None of the study participants developed any clinical evidence of autoimmunity. One patient developed a cardiomyopathy 18 months after DC vaccination. This was deemed to be related to the previous cisplatin treatment.

\section{Treg numbers}

After the first dosage of metronomic CTX, the percentage of Tregs of total CD4 cells significantly decreased from 9.43 (range $4.34-26.10$ ) to 4.51 (range 0.27 10.30) $(p=0.02)($ figure 4$)$. The initial percentage of Tregs was elevated in 4 patients, 2 surgical and 2 non-surgical, In one of these non-surgical patients, the level of Tregs was $28 \%$ of all CD4 cells. In 3 of these 4 patients, levels of Tregs decreased after seven days of low-dose CTX. There was no correlation between initial Treg levels or decrease and radiological response or survival. Also, no clear difference in Treg levels or decrease was established between surgical and nonsurgical patients in this small patient cohort.

\section{Immune responses}

In all patients, a comparison of pre-first-vaccine and post-third-vaccine serum samples showed a significant increase of antibodies reactive to the model antigen 
$\mathrm{KLH}$, both of the immunoglobulin ( $\mathrm{lg}) \mathrm{G}$ and IgM isotype. No or very low numbers of $\mathrm{KLH}$-specific antibodies were detected in undiluted serum of all patients before vaccination, illustrating the suitability of this antigen to determine the immune competence of the vaccine. Responses to $\mathrm{KLH}$ increased after the initial 3 vaccinations were completed (data not shown). Three months after the third vaccination, $\mathrm{KLH}$ responses were evidently present in the 6 patients in whom it could be determined. This proves that a successful immune reaction was induced by the DC vaccinations (Figure 5).

\section{Skin DTH testing}

The DTH skin test was performed two weeks after the third vaccination and was evaluable in all patients. There were no skin reactions to saline as the negative control. All patients revealed a positive test (erythema and/or induration) to the control antigen tetanus toxoid as well as to $\mathrm{KLH}(5 \mu \mathrm{g})$, and to $\mathrm{DC}$ with $\mathrm{KLH}$ ( $5 \times 10^{6}$ cells). This test proves that an effective immune response against $\mathrm{KLH}$ can be induced by DC vaccination. Eight patients tested positive to tumor lysate loaded DC $\left(5 \times 10^{6}\right.$ cells $)$.

In 6 patients, there was sufficient tumor material left for a post vaccination skin test with solely autologous tumor lysate $(10 \mu \mathrm{g})$, and 2 of these patients developed a positive DTH test. The 2 patients that revealed a negative skin test to both tumor lysate and tumor lysate loaded DCs were the patients with progressive disease after DC vaccination and the shortest survival. 


\section{Discussion}

In this study, we showed that combination treatment with CTX and DC immunotherapy is safe and feasible as a maintenance treatment in patients with mesothelioma after chemotherapy. The endpoint of the study was met with a significant decrease of Treg percentage of CD4 cells in blood of patients with increased levels. Radiographic tumor responses could not be established in all patients, as no measurable disease was present in the 5 surgical patients at inclusion in the trial. In the 5 non-surgical patients, one radiographic partial response was found. Overall disease control was found in 8 of the 10 patients, with 2 patients showing progression of disease. Overall survival was promising given the poor prognosis of this patient population. Considering the methodological flaws in this kind of comparison, overall survival was superior to a historical control group (data not shown), and also to published data(14-16).

The role of surgery in mesothelioma is under debate $(17,18)$. This is due to disease recurrences after surgery. Therefore, an effective adjuvant treatment is of the utmost importance. Animal models have shown that resection of an established primary tumor reduced the tumor-associated immune suppressive environment(19). This theoretically supports the combination of surgery and immunotherapeutic strategies. Our data confirm that this combination is feasible and should be studied further.

The addition of CTX resulted in a decrease in the percentage Tregs of total blood CD4 cells in most patients during CTX treatment, independent of the initial Treg percentage. This reduction was also found in previous studies with a similar 
metronomic low-dose schedule(20, 21). By contrast, single dose and twice-aweek dosing of CTX did not show an effect on Treg numbers(22, 23). Whether the decrease in Treg percentage caused an increased immune response cannot be concluded from these data, as no control group was included. The results found in this study are similar to our earlier murine experiments with DC immunotherapy and CTX(11). In that study, we did find survival to be increased when Treg levels were reduced.

The fact that we found normal peripheral blood Treg percentages in a significant number of patients may be a reflection of patient selection, since we selected patients who were non-progressing or responding to chemotherapy. It cannot be determined from our data whether Treg levels do play a role in these patients. It cannot be excluded that in the patients with normal Treg other immunosuppressive mechanisms are more prominent, such as M2 tumorassociated macrophages (TAMs) or myeloid derived suppressor cells (MSDCs). This finding could thus be of importance for personalizing immunotherapy. In this small study, no relations were established between initial Treg percentage or decrease in Tregs and radiologic response and overall survival. This can be due to the small sample size, but it is also possible that the number of circulating Tregs is not indicative of the Treg numbers within the tumor.

The DTH skin test proves that an effective immune response to $\mathrm{KLH}$ can be induced by DC vaccination, but this test was not positive for tumor lysate and/or tumor lysate loaded DCs in all patients. Although the number of patients is too small to draw any definite conclusion, the 2 patients that experienced progressive disease after DC vaccination and had the shortest survival had 
shown a negative skin test for both tumor lysate and tumor lysate loaded DCs. Therefore, the skin test should be evaluated in a larger cohort of patients as a marker for the outcome of DC vaccination therapy.

Serum KLH responses were seen in all patients, but only after completion of the third vaccination. In our previous trial, responses were already present after the first vaccination(5). This difference is likely due to the suppressive effect of CTX on B cells(24). This is subject for further study.

Another subject for further research is the impact of the immunosuppressive environment created by the tumor. This has now been shown to be both complex and subject to dynamic changes over time. Immune checkpoint pathways like CTLA-4 and PD-1 pathways negatively influence the immune response, and anti-CTLA4 antibodies showed clinical efficacy in patients with mesothelioma(25). Theoretically there might be a role for combining DC immunotherapy with these antibodies to increase the number of responding patients. However, other studies as well as our own have shown that the immune system is negatively impacted by immunosuppressive cells other than Tregs, such as M2 TAMs and MDSCs. These immunosuppressive cells negatively interact with checkpoint inhibitory therapy and with DC treatment(26, 27). Theoretically, decreasing the number of these cells may enhance the efficacy of checkpoint inhibition and DC treatment.

This study has some limitations. First, the number of patients included in this study is rather limited due to the considerable costs and labor involved in the production of the DC vaccination therapy. Furthermore, our study group was a heterogeneous group with different subhistologies, different stages of disease 
and different initial therapeutic approaches (e.g. surgical and non-surgical cases). Also, due to lack of randomization, the possibility of selection bias was present. While this did not seem to have an effect on the primary outcome of our study (Treg reduction and feasibility), it could have had an effect on the survival data.

Second, while radiographic CT scanning was performed, no PET scans were done. An earlier trial with immunotherapy showed a decrease in tumor FDG uptake(28, 29). Although the immune responses generated could also increase FDG-activity via the influx of immune cells, as has been suggested recently(30), PET-imaging could have yielded additional information.

Third, in the non-surgery group the autologous tumor lysate used for antigen loading of dendritic cells originated from treatment-naïve patients, while in the surgery group the tumor lysate was prepared from resected material derived from patients who had received chemotherapy as an induction to their surgery, which means that the tumor had already been treated. Three patients in the surgical group showed a prolonged survival, but selection bias should be considered. In addition, the 2 other surgical patients showed PD. The numbers of our study are too small to draw conclusions on this subject, but this will be addressed in future studies.

Fourth, the efficacy of CTX on the suppression of Tregs was assessed by measuring the Treg values in peripheral blood. While this way of studying Tregs is the most convenient method for the patient, Treg levels in peripheral blood might not be representative for the Treg density at the tumor site(31, 32). However, in mesothelioma, repeat biopsies are only feasible in selected patients. 
Fifth, although unlikely, we cannot rule out the possibility that leukapheresis has a temporary influence on the Treg numbers. Baseline values of Tregs were obtained at screening, before leukapheresis, while the Treg samples after CTX treatment were obtained after leukapheresis. To our knowledge, the impact of leukapheresis on Treg count is unknown. In 4 patients treated with DC vaccination, a total blood count including leucocyte differentiation was available at the 2 time points at which Tregs were measured, and these showed no change in leucocyte count or shift in leucocyte differentiation. Also, CTX was able to reduce Treg counts in previous studies without leukapheresis attributing the Treg decrease to CTX alone $(9,21)$.

\section{Conclusions of the study}

Short-term (1 week) treatment with a low-dose of CTX significantly reduced the mean Treg percentage of the total CD4 cells in peripheral blood, independent of initial Treg numbers. No correlation could be detected between the decrease in Treg and the response or overall survival. DC vaccination therapy in combination with low dose metronomic CTX is safe and feasible in patients with mesothelioma and no significant adverse effects were observed. Also, DC vaccination therapy proved to be feasible after debulking surgery. DC vaccination therapy combined with CTX resulted in radiographic disease control in 8 of the 10 patients. Overall survival was promising, with 7 out of 10 patients surviving $\geq 24$ months and two 
patients still alive 50 and 66 months after diagnosis. These outcomes warrant further study of this form of therapy in a larger, controlled trial. 


\section{Acknowledgements}

We thank Eric Braakman and Anita Schelen from the Department of Hematology for their excellent support on facility and ATMP regulations. Meriam Grootes and others are thanked for apheresing the patients and Femke Muskens for her technical assistance during DC culturing and phenotyping. We also thank Arnold Vulto, Valentina Lorenzi, and Chris Vermaat for their expert-advice and quality control of the DC vaccines. 


\section{References}

1. Nakas A, von Meyenfeldt E, Lau K, Muller S, Waller D. Long-term survival after lung-sparing total pleurectomy for locally advanced (International Mesothelioma Interest Group Stage T3-T4) non-sarcomatoid malignant pleural mesothelioma. Eur J Cardiothorac Surg 2012;41:1031-1036.

2. Linton A, Pavlakis N, O'Connell R, Soeberg M, Kao S, Clarke S, Vardy J, van Zandwijk N. Factors associated with survival in a large series of patients with malignant pleural mesothelioma in New South Wales. Br J Cancer 2014;111:1860-1869.

3. Baumann F, Flores E, Napolitano A, Kanodia S, Taioli E, Pass H, Yang H, Carbone M. Mesothelioma patients with germline BAP1 mutations have 7-fold improved long-term survival. Carcinogenesis 2015;36:76-81.

4. Cornelissen R, Heuvers ME, Maat AP, Hendriks RW, Hoogsteden HC, Aerts JGJV, Hegmans JPJJ. New Roads Open Up for Implementing Immunotherapy in Mesothelioma. Clinical and Developmental Immunology 2012;1-13.doi:10.1155/2012/927240.

5. Hegmans JP, Veltman JD, Lambers ME, de Vries IJM, Figdor CG, Hendriks RW, Hoogsteden HC, Lambrecht BN, Aerts JG. Consolidative dendritic cell-based immunotherapy elicits cytotoxicity against malignant mesothelioma. Am J Respir Crit Care Med 2010;181:1383-1390.

6. Hegmans JP, Aerts JG. Immunomodulation in cancer. Current Opinion in Pharmacology 2014;17:17-21.

7. Hegmans JPJJ, Hemmes A, Hammad H, Boon L, Hoogsteden HC, Lambrecht BN. Mesothelioma environment comprises cytokines and T-regulatory cells that suppress immune responses. Eur Respir J 2006;27:1086-1095. 8. Ireland DJ, Kissick HT, Beilharz MW. The Role of Regulatory T Cells in Mesothelioma. Cancer Microenviron 2012;5:165-172.

9. Ghiringhelli F, Menard C, Puig PE, Ladoire S, Roux S, Martin F, Solary E, Le Cesne A, Zitvogel L, Chauffert B. Metronomic cyclophosphamide regimen selectively depletes CD4+CD25+ regulatory $T$ cells and restores $T$ and NK effector functions in end stage cancer patients. Cancer Immunol Immunother 2007;56:641-648.

10. Kan S, Hazama S, Maeda K, Inoue Y, Homma S, Koido S, Okamoto M, Oka M. Suppressive effects of cyclophosphamide and gemcitabine on regulatory T-cell induction in vitro. Anticancer Res 2012;32:5363-5369.

11. Veltman JD, Lambers MEH, van Nimwegen M, de Jong S, Hendriks RW, Hoogsteden HC, Aerts JGJV, Hegmans JPJJ. Low-dose cyclophosphamide synergizes with dendritic cell-based immunotherapy in antitumor activity. $J$ Biomed Biotechnol 2010;2010:798467.

12. Aerts JG, Cornelissen R, Lambers MEH, Hoogsteden HC, Hegmans JPJJ. Dentritic Cell Based Immunotherapy in Combination with Metronomic Cyclophosphamide in Patients with Mesothelioma. iMig, Boston. 2012;

13. Byrne MJ, Nowak AK. Modified RECIST criteria for assessment of response in malignant pleural mesothelioma. Ann Oncol 2004;15:257-260. 
14. Ceresoli GL, Zucali PA, De Vincenzo F, Gianoncelli L, Simonelli M, Lorenzi E, Ripa C, Giordano L, Santoro A. Retreatment with pemetrexed-based chemotherapy in patients with malignant pleural mesothelioma. Lung Cancer 2011;72:73-77.

15. Zucali PA, Simonelli M, Michetti G, Tiseo M, Ceresoli GL, Collovà E, Follador A, Lo Dico M, Moretti A, De Vincenzo F, Lorenzi E, Perrino M, Giordano L, Farina G, Santoro A, Garassino M. Second-line chemotherapy in malignant pleural mesothelioma: results of a retrospective multicenter survey. Lung Cancer 2012;75:360-367.

16. Bearz A, Talamini R, Rossoni G, Santo A, de Pangher V, Fasola G, Rosetti F, Favaretto A, Gregorc V, Berretta M, others. Re-challenge with pemetrexed in advanced mesothelioma: a multi-institutional experience. BMC research notes 2012;5:482.

17. Treasure $T$, Utley M. Ten traps for the unwary in surgical series: a case study in mesothelioma reports. J Thorac Cardiovasc Surg 2007;133:1414-1418. 18. Bovolato $P$, Casadio $C$, Billè A, Ardissone F, Santambrogio L, Ratto GB, Garofalo G, Bedini AV, Garassino M, Porcu L. Does Surgery Improve Survival of Patients with Malignant Pleural Mesothelioma?: A Multicenter Retrospective Analysis of 1365 Consecutive Patients. Journal of Thoracic Oncology 2014;9:390396.

19. Broomfield S, Currie A, van der Most RG, Brown M, van Bruggen I, Robinson BWS, Lake RA. Partial, but not complete, tumor-debulking surgery promotes protective antitumor memory when combined with chemotherapy and adjuvant immunotherapy. Cancer Res 2005;65:7580-7584.

20. Alfaro C, Perez-Gracia JL, Suarez N, Rodriguez J, Fernandez de Sanmamed M, Sangro B, Martin-Algarra S, Calvo A, Redrado M, Agliano A, Gonzalez A, Rodriguez I, Bolaños E, Hervás-Stubbs S, Perez-Calvo J, Benito A, Peñuelas I, Vigil C, Richter J, Martinez-Forero I, Melero I. Pilot clinical trial of type 1 dendritic cells loaded with autologous tumor lysates combined with GM-CSF, pegylated IFN, and cyclophosphamide for metastatic cancer patients. J Immunol 2011;187:6130-6142.

21. Ge Y, Domschke C, Stoiber N, Schott S, Heil J, Rom J, Blumenstein M, Thum J, Sohn C, Schneeweiss A, Beckhove P, Schuetz F. Metronomic cyclophosphamide treatment in metastasized breast cancer patients: immunological effects and clinical outcome. Cancer Immunol Immunother 2012;61:353-362.

22. Ellebaek E, Engell-Noerregaard L, Iversen TZ, Froesig TM, Munir S, Hadrup SR, Andersen MH, Svane IM. Metastatic melanoma patients treated with dendritic cell vaccination, Interleukin-2 and metronomic cyclophosphamide: results from a phase II trial. Cancer Immunol Immunother 2012;61:1791-1804.

23. Chu CS, Boyer J, Schullery DS, Gimotty PA, Gamerman V, Bender J, Levine BL, Coukos G, Rubin SC, Morgan MA, Vonderheide RH, June CH. Phase I/II randomized trial of dendritic cell vaccination with or without cyclophosphamide for consolidation therapy of advanced ovarian cancer in first or second remission. Cancer Immunol Immunother 2012;61:629-641.

24. Sistigu A, Viaud S, Chaput N, Bracci L, Proietti E, Zitvogel L. Immunomodulatory effects of cyclophosphamide and implementations for vaccine 
design. Semin Immunopathol 2011;33:369-383.

25. Calabrò L, Morra A, Fonsatti E, Cutaia O, Amato G, Giannarelli D, Di Giacomo AM, Danielli R, Altomonte M, Mutti L, Maio M. Tremelimumab for patients with chemotherapy-resistant advanced malignant mesothelioma: an open-label, single-arm, phase 2 trial. Lancet Oncol 2013;14:1104-1111.

26. Wing JB, Ise W, Kurosaki T, Sakaguchi S. Regulatory T Cells Control Antigen-Specific Expansion of Tfh Cell Number and Humoral Immune Responses via the Coreceptor CTLA-4. Immunity 2014;41:1013-1025.

27. Noy R, Pollard JW. Tumor-Associated Macrophages: From Mechanisms to Therapy. Immunity 2014;41:49-61.

28. Torizuka T, Zasadny KR, Kison PV, Rommelfanger SG, Kaminski MS, Wahl RL. Metabolic response of non-Hodgkin's lymphoma to 131/-anti-B1 radioimmunotherapy: evaluation with FDG PET. J Nucl Med 2000;41:999-1005. 29. Chaise C, Itti E, Petegnief Y, Wirquin E, Copie-Bergman C, Farcet J-P, Delfau-Larue M-H, Meignan M, Talbot J-N, Molinier-Frenkel V. [F-18]-Fluoro-2deoxy-D: -glucose positron emission tomography as a tool for early detection of immunotherapy response in a murine B cell lymphoma model. Cancer Immunol Immunother 2007;56:1163-1171.

30. Gilles R, de Geus-Oei L-F, Mulders PFA, Oyen WJG. Immunotherapy response evaluation with 18F-FDG-PET in patients with advanced stage renal cell carcinoma. World J Urol 2013;31:841-846.

31. Nishikawa H, Sakaguchi S. Regulatory T cells in cancer immunotherapy. Curr Opin Immunol 2014;27C:1-7.

32. Tarhini AA, Edington H, Butterfield LH, Lin Y, Shuai Y, Tawbi H, Sander C, Yin Y, Holtzman M, Johnson J, Rao UNM, Kirkwood JM. Immune Monitoring of the Circulation and the Tumor Microenvironment in Patients with Regionally Advanced Melanoma Receiving Neoadjuvant Ipilimumab. In: Chatenoud L, editor. PLOS ONE 2014;9:e87705.

33. Sterman DH, Recio A, Haas AR, Vachani A, Katz SI, Gillespie CT, Cheng G, Sun J, Moon E, Pereira L, Wang X, Heitjan DF, Litzky L, June CH, Vonderheide RH, Carroll RG, Albelda SM. A Phase I Trial of Repeated Intrapleural Adenoviral-mediated Interferon- $\beta$ Gene Transfer for Mesothelioma and Metastatic Pleural Effusions. Mol Ther 2010;18:852-860.

34. Berger TG, Strasser E, Smith R, Carste C, Schuler-Thurner B, Kaempgen E, Schuler G. Efficient elutriation of monocytes within a closed system (Elutra) for clinical-scale generation of dendritic cells. J Immunol Methods 2005;298:61-72. 


\section{Tables}

\begin{tabular}{|c|c|c|c|c|c|}
\hline Patient No. & Gender & Age & Tumor subtype & TNM stage & ECOG ps ${ }^{\mathrm{c}}$ \\
\hline 1 & Male & 62 & Epithelioid & T1bN2M0 & 0 \\
\hline 2 & Female & 55 & Biphasic $^{* *}$ & T2NOMO & 0 \\
\hline 3 & Male & 63 & Epithelioid & T1bN0M0 & 1 \\
\hline 4 & Male & 71 & Epithelioid & T2NOMO & 0 \\
\hline 5 & Female & $35^{*}$ & Epithelioid & T1bN0M0 & 0 \\
\hline 6 & Male & 58 & Biphasic $^{* *}$ & T1bN0M0 & 1 \\
\hline 7 & Male & 48 & Epithelioid & T3N0MO & 0 \\
\hline 8 & Male & 78 & Epithelioid & T4N2M0 & 1 \\
\hline 9 & Male & 55 & Epithelioid & T1b0M0 & 0 \\
\hline 10 & Male & 75 & Epithelioid & T1aN0M0 & 1 \\
\hline
\end{tabular}

Table 1. Characteristics of the ten included MPM patients at time of diagnosis.

${ }^{a}$ Patient age in years; ${ }^{b}$ Histological tumor subtype by microscopic examination; ${ }^{c}$ WHO ps $=$ World Health Organisation performance status * Exposed as a child to high concentrations of asbestos by playing on a farmyard paved with asbestos. ${ }^{* *}$ Biphasic mesothelioma subtype is made up of both epithelioid cells and sarcomatoid cells. 


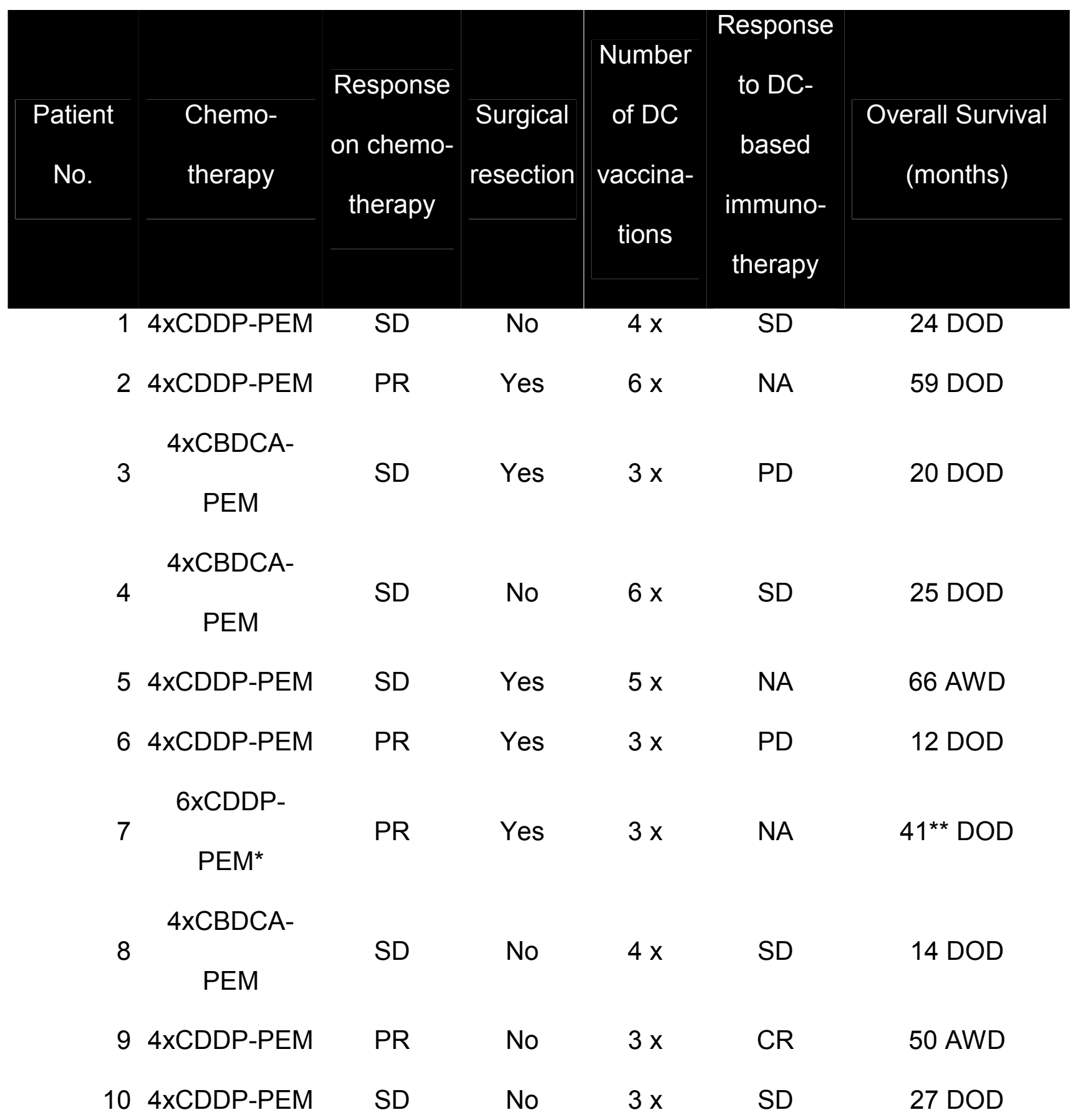

Table 2. Radiographic responses to chemotherapy and DC-based immunotherapy treatment, number of DC vaccinations and overall survival. $C D D P=$ cisplatin, $C B D C A=$ carboplatin,$P E M=$ pemetrexed 
$D O D=$ Died of disease $; A W D=$ Alive with disease (as of August 2015)

${ }^{*}$ Additional previous therapy prior to pleurectomy/decortication consisted of zoledronic acid, intrapleural gene therapy(33), gemcitabine with and without cisplatin alternating with cyclophosphamide.

** Survival post pleurectomy/decortication, OS after diagnosis was 83 months. 


$\begin{array}{lcccr} & \text { Grade } 1 & \text { Grade 2 } & \text { Grade } 3 & \text { Grade } 4 \\ \text { Fever } & 4 & 1 & 0 & 0 \\ \text { Nausea } & 4 & 0 & 0 & 0 \\ \text { Rash } & 3 & 0 & 0 & 0 \\ \text { Thoracic pain } & 3 & 0 & 0 & 0 \\ \text { Lethargy } & 3 & 0 & 0 & 0 \\ \text { Dyspnoea } & 2 & 0 & 0 & 0 \\ \text { Depression } & 0 & 1 & 0 & 0 \\ \text { Diarrhea } & 1 & 0 & 0 & 0 \\ \text { Cardiomyopathy } & 0 & 0 & 1 & 0 \\ \text { Abdominal pain } & 1 & 0 & 0 & 0\end{array}$

Table 3. Adverse events 


\section{Figures}

Figure 1: Schematic representation of the treatment procedure. $A 4^{\text {th }}$ and $5^{\text {th }}$ vaccination, after 6 or 12 months after the last DC vaccine, was given if enough dendritic cells were available.
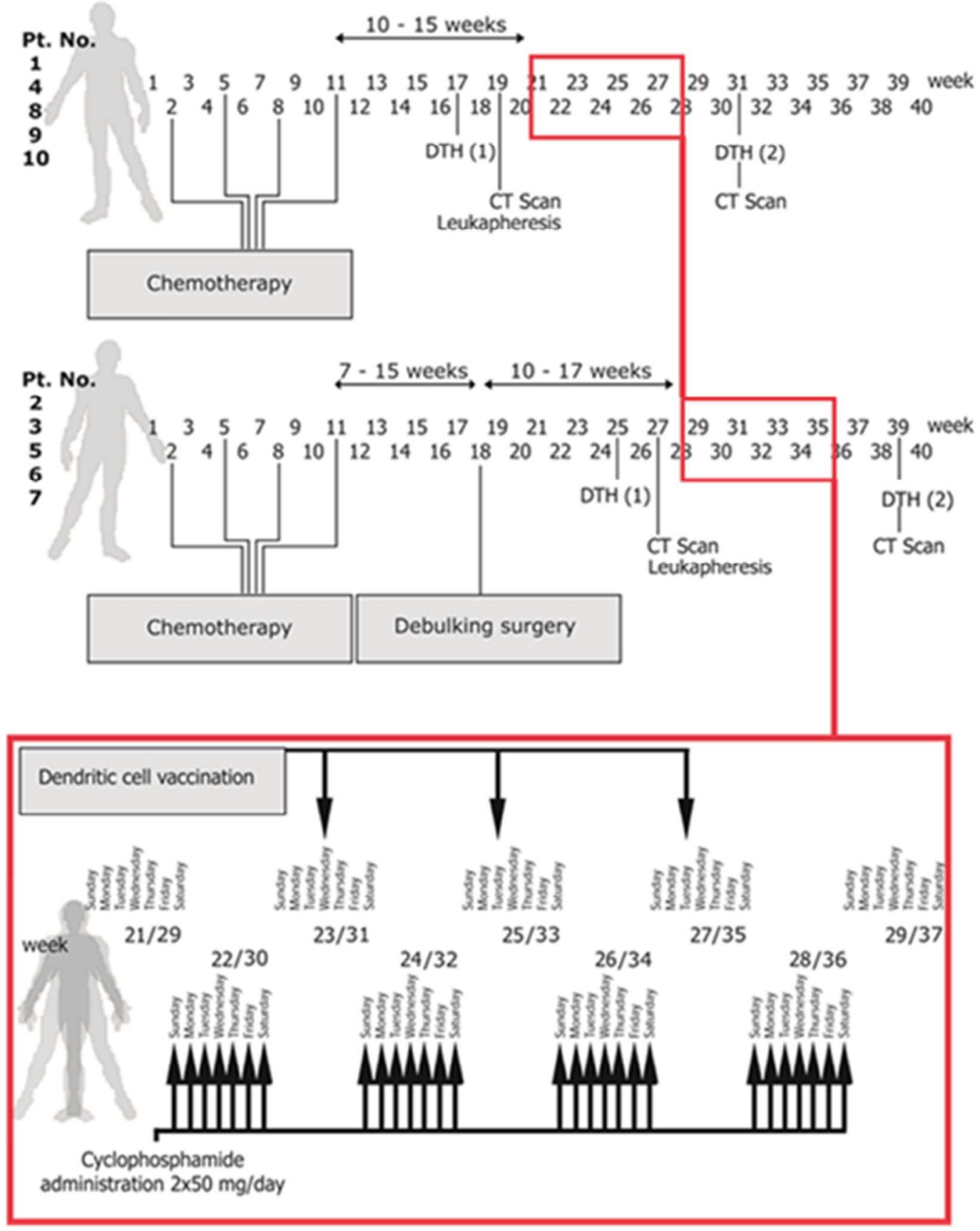
Figure 2. Kaplan-Meier plot of overall survival for all evaluable patients in the study. At the last follow-up on August 2015, 2 of 10 patients were still alive.

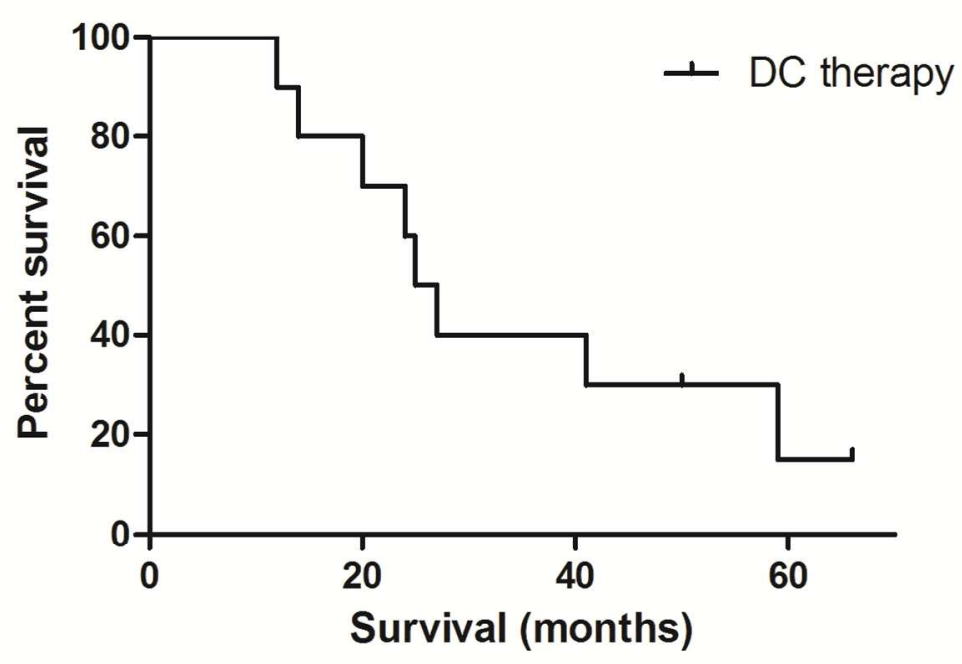


Figure 3. Sustained response in a surgical patient. (A) Before any treatment, two pleural masses are visible on the CT scan; one dorsally next to the spine and the other on the diaphragm. CT scan after chemotherapy showed stable disease (not shown). (B) After pleurectomy/decortication and DC administration, the cranial lesion is not visible and slight post-surgery abnormalities are visible. Caudally, due to post-surgery changes, it is difficult to detect any tumor. (C) 48 Months after DC treatment, no pleural masses are visible either cranially or caudally. 


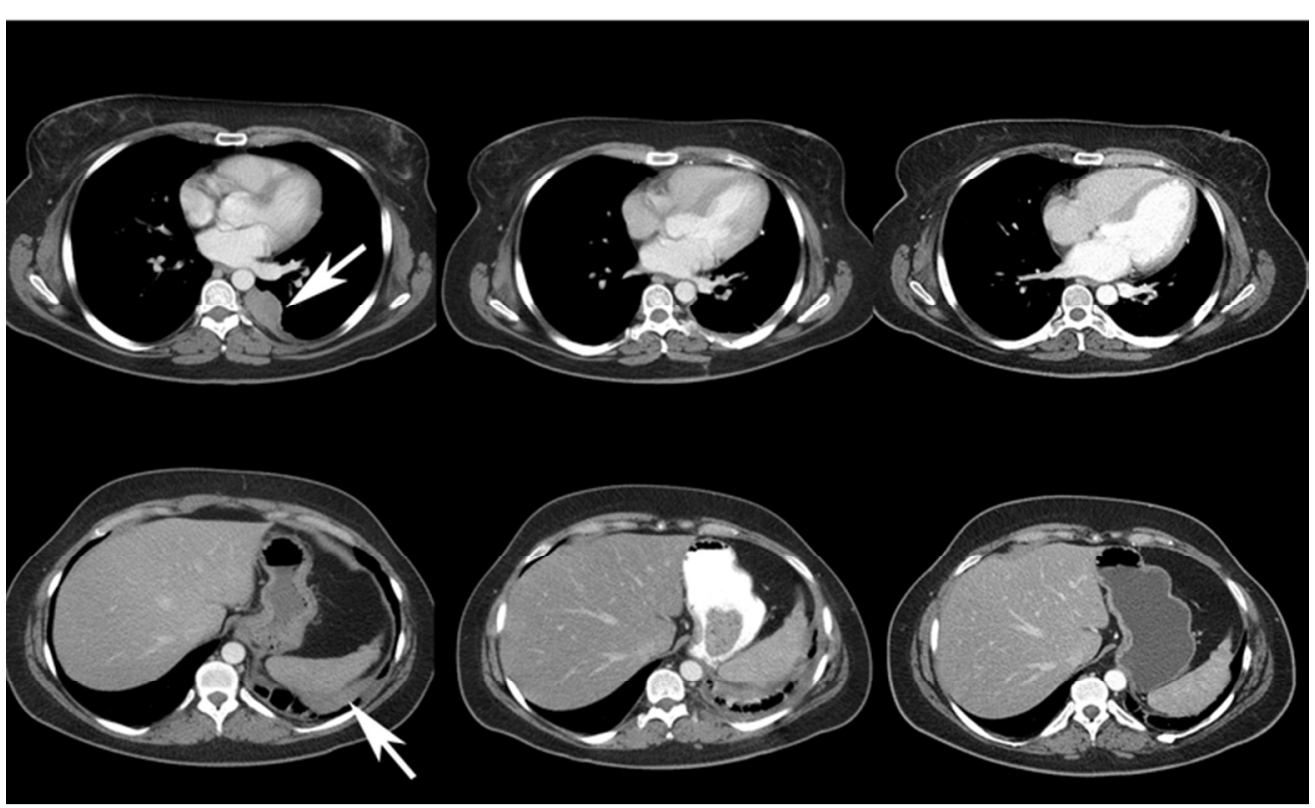

$31 \times 21 \mathrm{~mm}(600 \times 600$ DPI $)$ 
Figure 4. Measuring Tregs (CD3+CD4+CD25+FoxP3+CD127- cells) in blood samples of patients before CTX treatment and after the first week of metronomic CTX showed a significant decrease of the mean Treg percentage of total CD4 cells $(P=0.02)(A)$. (B) The individual Treg differences before and after the first CTX administration show a decrease in nine out of ten patients.

\section{Treg change after first CTX cycle}

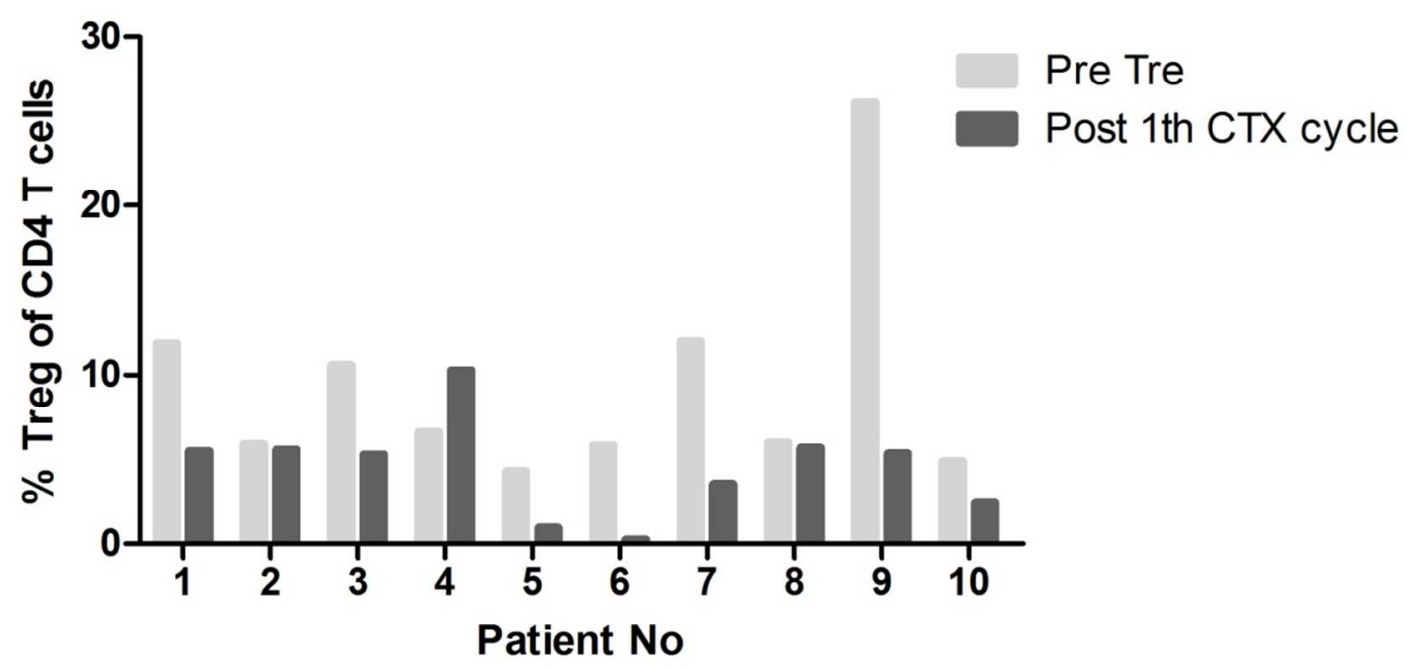


Figure 5. KLH-specific IgG increases after DC-based immunotherapy. Kinetics of IgG responses against $\mathrm{KLH}$ was measured in serially diluted serum of all patients during treatment and of seven patients following treatment. In patient 3 and 6 , no posttreatment is available due to the deteriorating clinical condition of these patients with progressive disease. In patient 7 , serum samples were unavailable at the specified time point because the patient was living abroad. Post-treatment increase of KLH specific IgG was evident in all measured samples. IgM levels (not shown) mirrored IgG levels. Pre Tre= four weeks before CTX / DC treatment; Post Tre $=3$ months after CTX / DC treatment.

\section{anti-KLH response in Serum (IgG)}

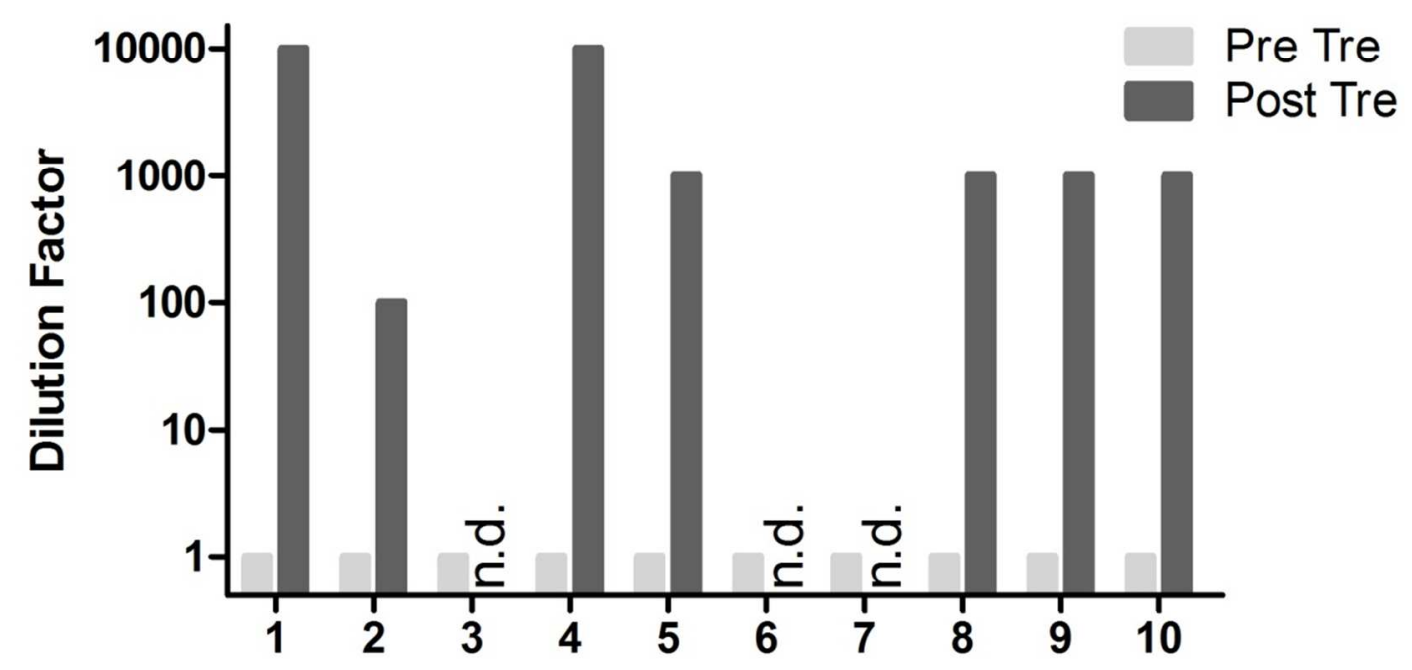

Patient No 


\section{Supplemental data}

Preparation of tumor lysate for DC loading

Isolation and preparation of autologous tumor lysate for DC loading was performed as described earlier(5). Tumor tissue (cases 2, 3, 5, 6, and 7) was removed by debulking surgery (pleurectomy/decortication), placed in phosphatebuffered saline (PBS) in sterile containers and transported immediately to the cleanroom facility. Representative tumor pieces were embedded in Tissue-Tek II optimum cutting temperature ([OCT] Miles, Naperville, IL, USA), snap-frozen and stored at $-80^{\circ} \mathrm{C}$. Remaining tumor tissue was dispersed to create a single cell suspension. In case of pleural effusions (cases 1, 4, 8, 9, and 10), fluid was aspirated and collected in sterile flasks without anticoagulant or other additives. Effusions were transported immediately to the cleanroom facility and centrifuged at $400 x g$ for $15 \mathrm{~min}$ at room temperature (RT). If necessary, small or large amounts of red blood cells in the cell pellet were removed by hypotonic lysis using sterile water or Ficoll-Paque PREMIUM, respectively. Cells were counted and resuspended at a concentration of $50 \times 106 / \mathrm{ml}$ in PBS. Cytospin preparations and/or tumor sections were prepared and examined for the presence of tumor cells using the following antibodies: cytokeratin 5/6, cytokeratin 19, thrombomodulin, N-cadherin, vimentin, HBME-1, calretinin, and Wilms' tumor 1 (WT-1) protein (all DAKO, Glostrup, Denmark). Total numbers of malignant cells exceeded $150 \times 10^{6}$ cells, and the percentage was at least $30 \%$ of total cells (inclusion criteria); in the case of biopsy material, total wet weight was at least 0.2 gram showing $>30 \%$ positivity for tumor markers. Remaining cells were lysed by 
six cycles of freezing in liquid nitrogen and thawing at room temperature followed by 100 Gy of irradiation. Large particles were removed by centrifugation ( $5 \mathrm{~min}$, 200xg), and supernatants were passed through a $0.45 \mu \mathrm{m}$ filter. The resulting tumor lysates were stored in aliquots at $-80^{\circ} \mathrm{C}$ until use.

\section{Dendritic cell culture}

We used our previously described method to generate clinical-grade mature dendritic cells in conformity with Good Manufacturing Practice (GMP) guidelines(5). In brief, concentrated 120 to $150 \mathrm{ml}$ leukocyte fractions were generated through a 4-h restricted peripheral blood leukapheresis, processing on average 9L of blood (COBE Spectra Apheresis System, Gambro BCT, Zaventem, Belgium). Peripheral blood mononuclear cells were then enriched using counterflow centrifugal elutriation (Elutra, Gambro BCT, Zaventem, Belgium) as described by Berger et al.(34). In $60 \%$ of the cases, the percentages of contaminating granulocytes in the enriched monocyte fractions after counter-flow centrifugal elutriation were below $10 \%$. A density gradient centrifugation was routinely performed on all samples to remove the granulocytes before starting the culturing process. In this way, the percentages of granulocytes at the start of the procedure were always below $8 \%$ for all preparations. Monocytes were resuspended at a concentration of $5 \times 10^{6}$ cells $/ \mathrm{ml}$ in XVIVO-15 (Cambrex Bio Science, Verviers, Belgium) supplemented with $2 \%$ pooled human AB serum (DC-culture medium [DC-CM]). The next day, half of the medium was removed and replaced by the same volume of DC-CM supplemented with $1000 \mathrm{IU} / \mathrm{ml}$ interleukin (IL)-4 (CellGenix, Freiburg, Germany) and $1600 \mathrm{IU} / \mathrm{ml}$ granulocyte 
macrophage-colony stimulating factor (GM-CSF; CellGenix). After 5 days of culture, semi-adherent and non-adherent cells were harvested by pipetting. Cells $\left(1 \times 10^{6}\right)$ were seeded per well of a 6 -well plate in fresh DC-CM supplemented with tumor cell lysate (1 tumor cell equivalent to 3 DC), $500 \mathrm{IU} / \mathrm{ml}$ IL-4, $800 \mathrm{IU} / \mathrm{ml}$ GMCSF, and $10 \mu \mathrm{g} / \mathrm{ml}$ keyhole limpet hemocyanin (KLH) (Calbiochem, La Jolla, CA, USA). The immune competence was monitored by means of co-loading with the protein $\mathrm{KLH}$, a foreign protein that stimulates T-helper responses. On day 8, the maturation cocktail was added (prostaglandin E2 [PGE2 Prostin E2; $10 \mu \mathrm{g} / \mathrm{ml}$ Pharmacia\&Upjohn, Puurs, Belgium], tumor necrosis factor-alpha [TNF- $\alpha, 20$ ng/ml), interleukin (IL)-1 $\beta$ [5 ng/ml], and IL-6 [15 ng/ml; all CellGenix]). Cells were harvested at day 10 and $50 \times 10^{6}$ cells were used for immediate vaccination; the remaining cells were cryopreserved in DMSO for later vaccinations $\left(55 \times 10^{6}\right.$ cells per vial) and for DTH skin testing. Loaded DC are defined as an advanced therapy medicinal product (ATMP) and released for vaccination after thorough check by accredited qualified person according to the Clinical Trials Directive (2001/20/EC). These include consistency and quality in the processing steps and final products and check of the manufacturing facility, among others. The vaccine was routinely analyzed for DC purity and tested for infectious agents before administration to patients. Several quality control tests were performed before the cellular vaccine was released.

Flow cytometric analysis of clinical-grade DC

An aliquot of the vaccine preparation was retained to examine the expression of extracellular markers. The following monoclonal antibodies were purchased from 
BD Biosciences / BD Pharmingen (Erembodegem, Belgium): FITC-conjugated CD86 and CD195, PE-conjugated CD83 and CD-95, CD80 - PE-Cy5, CD209 PerCP-Cy5.5, CD11c - APC, and APC-Cy7 conjugated HLA-DR. The specificity of the antibodies was checked using equivalent concentrations of fluorochrome- and isotype-matched negative control immunoglobulins. Cells were washed with FACS buffer (PBS supplemented with $0.25 \%$ BSA, $0.5 \mathrm{mM}$ EDTA, and $0.05 \%$ sodium azide) and counted. At least $0.4 \times 10^{6}$ cells in $100 \mu$ were stained with appropriate dilutions of antibodies. Cells were incubated on ice for 30 min in the dark, washed twice with FACS buffer and analysed by means of LSR flow cytometry (BD Biosciences). Release criteria for each batch of DC were sterility testing (negative for aerobic or anaerobic microorganisms), viability (>80\% viable by flowcytometry and propidium iodide or 7-aminoactinomycin D [7-AAD]), purity (>95\% CD11c ${ }^{+}$ $\mathrm{MHC}$ class $\mathrm{II}^{+}$), maturation (>60\% of CD80 expression) and stability after freezing.

\section{Delayed type hypersensitivity skin test}

To assess the patient's immunocompetence, DTH skin testing was performed one week before the apheresis by means of intradermal application of tetanus toxoid (positive control) and a physiological salt solution (negative control) on the ventral surface of the patient's forearm. If this did not result in a response to tetanus that could be related to the effects of chemotherapy, apheresis was postponed and the skin DTH test was repeated two weeks later. If the second test was also negative the patient was excluded from participation in the study. A DTH skin test was also performed two weeks after the third vaccination; it was further completed with 
autologous tumor lysate $(10 \mu \mathrm{g}), \mathrm{KLH}(5 \mu \mathrm{g})$, tumor lysate loaded DC with or without $\mathrm{KLH}$ (both $5 \times 10^{6}$ cells). DTH responses were evaluated after $48 \mathrm{~h}$.

Immune response assessment against $K L H$

Serum samples were collected into SST serum separation tubes (BD biosciences) before, during, and in the lifelong clinical follow-up of the patients. After allowing the serum $30 \mathrm{~min}$ to clot, tubes were centrifuged $10 \mathrm{~min}$ at $1000 \times \mathrm{g}$. Serum was collected, aliquoted and stored at $-80^{\circ} \mathrm{C}$ until use. Humoral responses to $\mathrm{KLH}$ were measured in the serum of patients by ELISA. Microtiter plates (96 wells) were coated overnight at $4{ }^{\circ} \mathrm{C}$ with $25 \mu \mathrm{g} / \mathrm{ml} \mathrm{KLH}$ in PBS per well. After blocking the plates with $1 \%$ powdered milk in PBS, different concentrations of patient serum (range, 1 in 100 to 1 in 500,000) were added for $1 \mathrm{~h}$ at room temperature. After extensive washing, specific anti-human IgG or anti-human IgM conjugated with horseradish peroxidase were allowed to bind for $1 \mathrm{~h}$ at room temperature. Peroxidase activity was revealed with the use of 3,3'5,5-tetramethyl-benzidine (TMB) as substrate, and absorbance was measured in a microtiter plate reader (VersaMax, Molecular Devices, Sunnyvale, CA, USA).

\section{Treg analysis}

Blood samples obtained before the treatment protocol and at the first vaccination (after induction cyclophosphamide) were analyzed. Tregs were defined as CD3+, CD4+, CD25+, FoxP3+, and CD127-. Before immune staining, the cells were stained for viability using LIVE/DEAD Fixable Aqua Dead Cell Stain (Invitrogen life technologies) in PBS. Subsequently the cells were washed in FACS buffer 
and incubated $30 \mathrm{~min}$ at $4^{\circ} \mathrm{C}$ with APC-eF780 labeled anti-CD3 (Clone UCHT1; eBioscience), AF700-labeled anti-CD4 (Clone RPA-T4; eBioscience), PE-Cy7labeled anti-CD25 (Clone MA251; BD Biosciences), V450-labeled anti-CD127 (Clone hIL7R-M2; BD Biosciences), PE-TexasRed-labeled anti-CD45RA (Clone MEM-56; Invitrogen life technologies), FITC-labeled anti-CCR7 (Clone 150503; R\&D Systems, Abingdon, UK) and BV605-labeled anti-CCR4 (Clone 1G1; BD Biosciences). Thereafter the cells were washed, fixated, and permeabilized using the eBioscience FoxP3 kit, and the cells were stained intracellularly with PElabeled anti-FoxP3 (Clone 236A/E7; eBioscience), APC-labeled anti-Ki-67 (Clone 20Raj1; eBioscience) and PerCP-eF710-labeled anti-CTLA-4 (Clone 14D3; eBioscience). Before analysis, the cells were washed with FACS buffer. The samples were measured on an LSR-II flow cytometer (BD Biosciences), and data analysis was performed using FlowJo software.

\section{Statistical analysis}

Mean Treg percentage was compared before and after CTX administration and $p$ values were calculated with the Wilcoxon signed-rank test. Statistical calculations were performed using IBM SPSS Statistics 21. Statistical significance was established at the $p<0.05$ level, and analysis was two-sided. 